\title{
Numerical implementation of a matter-wave Mach-Zehnder interferometer
}

\section{Implementación numérica de un interferómetro de Mach-Zehnder con ondas de materia}

\author{
Gustavo Marín-Alvarado, Karen Rodríguez-Ramírez* \\ Departamento de Física, Universidad del Valle, A.A. 25360, Cali, Colombia \\ (*) E-mail: karem.c.rodriguez@correounivalle.edu.co \\ Received / Recibido: 16/04/2018 Accepted / Aceptado: 26/09/2019 \\ DOI: $10.7149 /$ OPA.52.3.50309
}

\begin{abstract}
:
More than two decades ago a new state of matter called Bose-Einstein Condensation was proved experimentally although predicted long before in 1924-25. At extremely low temperatures and controlled densities, this exotic state can be achieved where all atoms share the same energy and linear momentum similarly as the light behaves in a laser. Therefore, it is interesting to analyze the matterwave counterparts for the nowadays well-known optical devices. Here, we present a numerical implementation of a Mach-Zehnder interferometer which we engineered by means of time-dependent external trapping potentials. We use an appropriate implementation of the finite-difference method for the spatial discretization and the Runge-Kutta technique for the time evolution. The dynamics of both conjugate variables, namely density and phase of the matter-wave functions are shown. Our aim is also to show that this kind of problems and techniques can be addressed in standard courses of Quantum Mechanics and Computational Physics.
\end{abstract}

Key words: Bose-Einstein condensates, nonlinear optics, atomic and molecular physics, coherent optical effects.

\section{RESUMEN:}

Hace más de dos décadas se realizó experimentalmente un nuevo estado de la materia, el Condensado de Bose-Einstein, predicho en 1924-25. A bajas temperaturas y densidades controladas, los átomos en este estado exótico comparten la misma energía y momento lineal al igual que lo hace la luz láser. Por tanto, es interesante analizar los dispositivos para ondas de materia análogos a los conocidos en óptica. En el presente trabajo se discute una implementación numérica de un interferómetro de Mach-Zehnder construido por medio de potenciales externos dependientes del tiempo. Se implementa apropiadamente el método de diferencias finitas para la discretización espacial y el método de RungeKutta para la temporal, obteniendo la dinámica de ambas variables conjugadas, la densidad y la fase de la onda de materia. Nuestro objetivo también es mostrar que esta clase de problemas y técnicas pueden ser tratados en los cursos estándar de mecánica cuántica y física computacional.

Palabras clave: Condensados de Bose-Einstein, óptica no lineal, física atómica y molecular, efectos ópticos coherentes.

\section{REFERENCES AND LINKS / REFERENCIAS Y ENLACES}

[1] A. Einstein Sitzungsberichte der Preussischen Akademie der Wissenschaften, 3, 18 (1925).

[2] S. Bose Zeitschrift für Physik A hadrons and Nuclei, 75, 3969 (1924).

[3] S. Chu, "Nobel lecture: The manipulation of neutral particles," Rev. Mod. Phys., vol. 70, pp. 685-706, Jul 1998. 
[4] C. N. Cohen-Tannoudji, “Nobel lecture: Manipulating atoms with photons," Rev. Mod. Phys., vol. 70, pp. 707-719, Jul 1998.

[5] W. D. Phillips, "Nobel lecture: Laser cooling and trapping of neutral atoms," Rev. Mod. Phys., vol. 70, pp. 721-741, Jul 1998.

[6] M. H. Anderson, J. R. Ensher, M. R. Matthews, C. E. Wieman, and E. A. Cornell, “Observation of boseeinstein condensation in a dilute atomic vapor," Science, vol. 269, no. 5221, pp. 198-201, 1995.

[7] K. B. Davis, M. O. Mewes, M. R. Andrews, N. J. Van Druten, D. S. Durfee, D. M. Kurn, and W. Ketterle, "Bose-Einstein condensation in a gas of sodium atoms," Phys. Rev. Lett., vol. 75, pp. 3969-3973, Nov 1995.

[8] A. J. Leggett, "Bose-Einstein condensation in the alkali gases: Some fundamental concepts," Rev. Mod. Phys., vol. 73, pp. 307-356, Apr 2001.

[9] F. Dalfovo, S. Giorgini, L. P. Pitaevskii, and S. Stringari, "Theory of Bose-Einstein condensation in trapped gases," Rev. Mod. Phys., vol. 71, pp. 463-512, Apr 1999.

[10] I. Bloch, J. Dalibard, and W. Zwerger, "Many-body physics with ultracold gases," Rev. Mod. Phys., vol. 80, pp. 885-964, Jul 2008.

[11] S. Giorgini, L. P. Pitaevskii, and S. Stringari, "Theory of ultracold atomic fermi gases," Rev. Mod. Phys., vol. 80, pp. 1215-1274, Oct 2008.

[12] S. Haroche, "Nobel Lecture: Controlling photons in a box and exploring the quantum to classical boundary", Rev. Mod. Phys., vol 85, pp. 1083, July 2013.

[13] F. D. Haldane, "Nobel Lecture: Topological quantum matter" Rev. Mod. Phys., vol 89, pp. 040502 , October 2017.

[14] D. Strickland, "Nobel Lecture: Generating high-intensity ultrashort optical pulses" Rev. Mod. Phys. Vol 91, pp. 030502, July 2019.

[15] T. Schumm, S. Hofferberth, L. M. Andersson, S. Wildermuth, S. Groth, I. Bar-Joseph, J. Schmiedmayer, and P. Kruger, "Matter-wave interferometry in a double well on an atom chip," Nat. Phys., vol. 1, pp. 57-62, Oct 2005.

[16] T. Berrada, S. van Frank, R. Bcker, T. Schumm, J.-F. Schaff, and J. Schmiedmayer, "Integrated MachZehnder interferometer for Bose-Einstein condensates," Nature Communications, vol. 4, p. 2077, June 2013.

[17] O. Morsch and M. Oberthaler, "Dynamics of Bose-Einstein condensates in optical lattices," Rev. Mod. Phys., vol. 78, pp. 179-215, Feb 2006.

[18] A. L. Fetter, “Rotating trapped Bose-Einstein condensates," Rev. Mod. Phys., vol. 81, pp. 647-691, May 2009.

[19] V. Zapf, M. Jaime, and C. D. Batista, "Bose-Einstein condensation in quantum magnets," Rev. Mod. Phys., vol. 86, pp. 563-614, May 2014.

[20] A. D. Cronin, J. Schmiedmayer, and D. E. Pritchard, "Optics and interferometry with atoms and molecules," Rev.Mod. Phys., vol. 81, pp. 1051-1129, Jul 2009.

[21] S. van Frank, A. Negretti, T. Berrada, R. Bcker, S. Montangero, J.-F. Schaff, T. Schumm, T. Calarco, and J. Schmiedmayer, "Interferometry with non-classical motional states of a Bose-Einstein condensate," Nature Communications, vol. 5, p. 4009, May 2014.

[22] W. Bao, D. Jaksch, and P. A. Markowich, "Numerical solution of the Gross-Pitaevskii equation for BoseEinstein condensation," Journal of Computational Physics, vol. 187, no. 1, pp. 318 - 342, 2003.

[23] E. P. Gross, "Structure of a quantized vortex in boson systems," Il Nuovo Cimento (1955-1965), vol. 20, no. 3, pp. 454-477, 1961.

[24] L. Pitaevskii, "Vortex lines in an imperfect Bose gas," Sov. Phys. JETP, vol. 13, no. 3, p. 451, 1961.

[25] A. Griffin, D. W. Snoke, and S. Stringari, Bose-Einstein Condensation. Cambridge University Press, 1995. 


\section{Introduction}

Gases exhibiting full quantum mechanical behavior have been studied since 1925 when A. Einstein [1], based on the statistics developed by S. Bose [2], predicted a new state of matter, the so-called Bose-Einstein Condensate (BEC). In 1970, several methods to cool and trap atoms with laser light were developed, for which S. Chu [3], C. Cohen-Tannoudji [4] and W. Phillips [5] were awarded with the Nobel prize in 1997. Based on these techniques, in 1995 the BEC was experimentally achieved by C. Wieman and E. Cornell [6] and W. Ketterle [7], thus, awarded with the Nobel prize in 2001.

One of the most fascinating features of working with these condensates is the ability to precisely manipulate a macroscopic and extremely cold object $(\sim 10-6 \mathrm{~K})$ and yet continue to speak of a quantum system. The reason comes from the fact that the BEC has millions of identical atoms sharing the same quantum state ( 10-4 atoms per cubic centimeter) [8], and it is large enough to be optically imaged.

Ultracold atoms trapped optically and/or magnetically constitute an extraordinary tool for the analysis of coherent matter under extremely well-controlled conditions as highlighted by the achievement of the BEC and the "super-atom"-properties it exhibits [9]. The field has been growing fast due to the novel possibilities it offers for studies on fundamental quantum-mechanical processes. High research activity has been reported in the last two decades including experimental, theoretical and numerical developments, such that multiple Nobel prices still been awarded in this field during the last two decades [10-14].

The particle-wave duality of the BEC as a macroscopic quantum object allows us to use it for the realization of matter-wave interferometers [15]. Furthermore, quantum interference constitutes a major challenge for testing quantum mechanical foundations besides the realization of ultra-precise measurements. Thus, inspired on the advanced manipulation of atomic BECs [16-19], interferometers based on ultracold atoms attract great interest.

The development of atom optics counterparts to beam splitters, phase shifters and recombiners makes this field a complement to the optical interferometers [20-22]. In the present work, we implement a MachZehnder interferometer using a trapped Bose-Einstein condensate with a Gaussian profile. The incoming matter-wave is divided into two beams by a 50:50 beam splitter made by a time-dependent double well potential built with piecewise functions. One of the beams travels a longer distance carrying a phase difference with respect to the other beam. When the two matter-waves are recombined the phase difference is also registered as a density/phase pattern deformation. Both, splitting and recombination stages can be performed either smoothly (in an adiabatic process) or suddenly (in a non-adiabatic manner). Each of them strongly depends on the time it takes to be experimentally implemented leading to different study proposes.

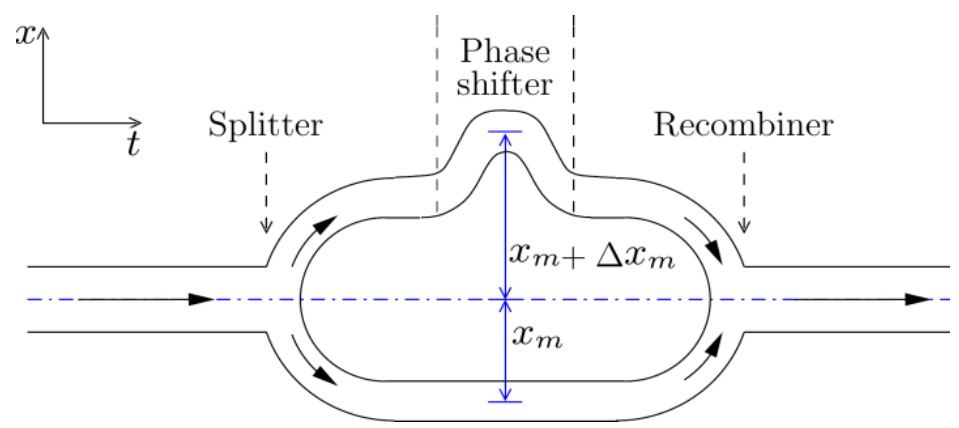

Fig.1. Sketch of the implemented Mach-Zehnder interferometer, where $\mathrm{x}_{\mathrm{m}}$ and $\mathrm{x} m+\Delta \mathrm{xm}$ denote the location of the trapping potential minima.

This paper is organized as follows: in Sect. 2 we describe the Gross-Pitaevskii equation to model the BEC. Section 3 is devoted to describe the developed time-dependent trapping potential and sect. 4 shows the numerical methods for the implementation of the interferometer. Two distinct regimes are here used and combined: i) the adiabatic regime, where the whole process is carried out in the most smoothly manner, ii) 
the non-adiabatic regime, where sudden changes are implemented. Density and phase dynamics of the matter-wave for three combinations of the regimes are presented in Sect. 5, and finally Sect. 6 concludes.

\section{Weakly interacting model}

In the subsequent treatment we follow W. Bao et al. [22] because, under our viewpoint, the explanation is clear enough to be understood in the undergraduate level. Here, we make a short overview in order to keep the document self-consistent. We are interested in study a bosonic cloud whose temperature is very close to the absolute zero, i.e. of the order of few micro-Kelvin $(\mu \mathrm{K})$. Under this circumstance, the system undergoes Bose-Einstein condensation and is described by a macroscopic wave function, where its time evolution is determined by a non-linear Schrödinger equation, called the Gross-Pitaevskii (GP) equation [23-25]. When the system is trapped using a harmonic confinement given by

$$
V_{e x t}=\frac{1}{2} m\left(w_{x}^{2} x^{2}+w_{y}^{2} y^{2}+w_{z}^{2} z^{2}\right)
$$

the GP-equation takes the form,

$$
i \hbar \frac{\partial \psi(\vec{x}, t)}{\partial t}=\left[\frac{-\hbar^{2}}{2 m} \nabla^{2}+V_{e x t}+N U_{0}\left|\psi(\vec{x}, t)^{2}\right|\right] \psi(\vec{x}, t)
$$

here $\vec{x}=(x, y, z)^{T}$ is the spatial-coordinates vector, $m$ is the atomic mass, $\hbar$ is the Planck constant divided by $2 \pi, N$ is the condensate atom-number, $\mathrm{w}_{\mathrm{x}}, \mathrm{w}_{\mathrm{y}}$ and $\mathrm{w}_{\mathrm{z}}$ are the trap frequencies in the $\mathrm{x}-\mathrm{y}$ - and $\mathrm{z}$-directions, respectively. The atomic interaction inside the condensate can be described by a contact potential of the form $U_{0}=\left(4 \pi \hbar^{2} a\right) / m$. Here $a$ is the isotropic s-wave scattering length, where for repulsive interactions $a>0$ and $a<0$ for attractive interactions. It is always necessary to ensure that the wave-function is normalized to the number of particles, $N$. Specifically, one requires,

$$
\int\left|\psi(\vec{x}, t)^{2}\right| d \vec{x}=N .
$$

\section{2.a. Dimensionless Gross-Pitaevskii equation}

The equation (2), under the normalization condition given by the equation (3), becomes dimensionless introducing the change of variables,

$$
\bar{t}=w_{x} t, \overline{\vec{x}}=\frac{\vec{x}}{x_{s}}, \bar{\psi}(\overline{\vec{x}}, \bar{t})=x_{s}^{3 / 2} \psi(\vec{x}, t),
$$

where $\mathrm{xs}_{\mathrm{s}}$ is the characteristic length of the condensate. Multiplying by the factor $1 /\left(m w_{x}^{2} x_{s}^{1 / 2}\right)$ and finally removing the bars on the variables, we end-up with the following dimensionless Gross-Pitaevskii equation,

$$
i \frac{\partial \psi(\vec{x}, t)}{\partial t}=\left[\frac{-\epsilon}{2} \nabla^{2}+V_{e x t}+g\left|\psi(\vec{x}, t)^{2}\right|\right] \psi(\vec{x}, t)
$$

where the parameters are

$$
\begin{aligned}
& \epsilon=\frac{\hbar}{w_{x} m x_{s}^{2}}=\left(\frac{a_{0}}{x_{s}}\right)^{2}, \quad V(\vec{x})=\frac{1}{2 \epsilon}\left(x^{2}+\gamma_{y}^{2} y^{2}+\gamma_{z}^{2} z^{2}\right) \\
& \delta=\frac{U_{0} N}{a_{0}^{3} \hbar w_{x}}=\frac{4 \pi a N}{a_{0}}, \quad \gamma_{y}=\frac{w_{y}}{w_{x}}, \gamma_{z}=\frac{w_{z}}{w_{x}}, g=\delta \epsilon^{3 / 2}, a_{0}=\sqrt{\frac{\hbar}{w_{x} m}}
\end{aligned}
$$

Here, $a_{0}$ is the harmonic-oscillator ground-state length in x-direction. We considered $w_{y, z} \ll w_{x}$, and therefore $\gamma_{y, z} \ll 1$ providing the effective confinement to be only in one-dimension. Hence, we retrieve the following dimensionless $1 \mathrm{D}$ Gross-Pitaevskii equation, 


$$
i \frac{\partial \psi(x, t)}{\partial t}=\left[\frac{-\epsilon}{2} \nabla^{2}+V_{e x t}(x)+g\left|\psi(x, t)^{2}\right|\right] \psi(x, t),
$$

here $V(x)=x^{2} / 2$. The initial state is chosen to have a Gaussian profile of the form,

$$
\psi(x, t=0)=B \exp \left[-\left(x-x_{0}\right)^{2} /\left(2 \sigma^{2}\right)\right]
$$

where $\mathrm{B}$ is the amplitude and measures the height of the profile determined by the normalization, $x_{0}=0$ is the center of the distribution and $\sigma$ controls the width of the "bell" and gives us the standard deviation.

\section{Dynamically driven external trapping potential}

We load the BEC on a time-dependent external trapping potential, i.e. in equation (7), the potential is allowed to change on time, $V(x, t)$, either in an adiabatic or non-adiabatic way. Furthermore, symmetric and non-symmetric configurations respect to the center of the initial single well, $x=0$, are also explored. A diagram of the analyzed Mach-Zehnder scheme is presented in figure (1). The time-dependent trapping potential has the form,

$$
V(x, t)=A(x-q(x, t))^{2}\left(x+q^{\prime}(x, t)\right)^{2}
$$

Here the functions $q(x, t)$ and $q^{\prime}(x, t)$ have been chosen conveniently for each desired case. Note that at $t=0$ the single well has a quadratic profile, see figure 2(a). In such way, the time-dependent symmetric trapping potential $(\mathrm{S})$, in the adiabatic regime (Ad), is achieved by the follow piecewise function,

$$
V_{S, A d}(x, t)=\left\{\begin{array}{c}
V_{1}\left(x, t, t_{1}\right) \forall x, t_{0} \leq t<t_{1} \\
V_{2}(x) \forall x, t_{1} \leq t<t_{2} \\
V_{1}\left(x, t, t_{2}\right) \forall x, t_{2} \leq t<t_{3}
\end{array}\right.
$$

Whereas the non-symmetric time-dependent potential or Mach-Zehnder trap (MZ), also in the adiabatic regime, is given by

$$
V_{M Z, A d}(x, t)=\left\{\begin{array}{c}
V_{1}\left(x, t, t_{1}\right) \forall x, t_{0} \leq t<t_{1} \\
V_{2}(x) x \leq 0, t_{1} \leq t<t_{2} \\
V_{M Z}\left(x, t, t_{M Z}\right) x>0, t_{1} \leq t<t_{2} \\
V_{1}\left(x, t, t_{2}\right) \forall x, t_{2} \leq t<t_{3}
\end{array}\right.
$$

The $V_{M Z, A d}(x, t)$ trap presents a discontinuity at $x=0$ but this fact does not affect our numerical experiment since the population is already distributed in the two separated-wells, see figures 2(b) and 2(c).

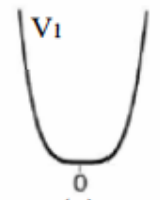

(a)

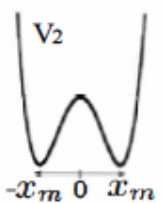

(b)

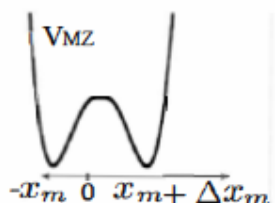

(c)

Fig.2.Snapshots of the three types of time-dependent external potentials: (a) the single-well $V_{1}\left(x, t, t_{i}\right)$, (b) the symmetric doublewell $V_{2}(x)$, (c) non-symmetric double-well or Mach-Zehnder trap $V_{M Z}\left(x, t, t_{M Z}\right)$.

The sketches of the functions $\mathrm{V}_{1}, \mathrm{~V}_{2}$ and $\mathrm{V}_{\mathrm{Mz}}$ are presented in figure 2 and their definitions are as follows, 


$$
\begin{gathered}
V_{1}\left(x, t, t_{i}\right)=A\left(x-\sqrt{x_{0}^{2}-x_{0}^{2}\left(t-t_{i}\right)^{2}}\right)^{2}\left(x+\sqrt{x_{0}^{2}-x_{0}^{2}\left(t-t_{i}\right)^{2}}\right)^{2}, \\
V_{2}(x)=A\left(x-x_{0}\right)^{2}\left(x+x_{0}\right)^{2}, \\
V_{M Z}\left(x, t, t_{M Z}\right)=A\left(x-\frac{x_{0}}{2} e^{\frac{-\left(t-t_{M Z}\right)^{2}}{\sigma}}-\frac{x_{0}}{2}\right)^{2}\left(x-\frac{x_{0}}{2} e^{\frac{-\left(t-t_{M Z}\right)^{2}}{\sigma}}+\frac{x_{0}}{2}\right)^{2} .
\end{gathered}
$$

\section{Numerical implementation}

In order to solve the equation (7) using the potentials (9) and (10), we use a straightforward discretization for both space and time coordinates. First, let us discuss the spatial dependency. We discretized the derivatives using the finite central difference approximation,

$$
\delta[\psi](x)=\psi\left(x+\frac{\Delta x}{2}\right)-\psi\left(x-\frac{\Delta x}{2}\right),
$$

where $\Delta x$ is the space-step. Therefore, the second derivative of the wave-function is expressed as

$$
\psi^{\prime \prime}(x) \sim \frac{\delta^{2}[\psi](x)}{\Delta x^{2}}=\frac{\psi(x+\Delta x)-2 \psi(x)+\psi(x-\Delta x)}{\Delta x^{2}} .
$$

The time-dependent partial differential equation (7) can be written in a general fashion as

$$
\frac{\partial \psi}{\partial t}=f(x, t, \psi)
$$

The complex function $\mathrm{f}$ contains the spatial discretization, hence boundary conditions. For a fixed time $t$, three separated cases must be considered,

$$
f(x, t, \psi)=\left\{\begin{array}{c}
f_{\text {Left }} x=-L / 2 \\
f_{\text {Default }}-L / 2<x<L / 2 \\
f_{\text {Right }} x=L / 2,
\end{array}\right.
$$

here $L$ is the size of the numerical calculation box. The function $f_{\text {Left }}$ ( $\left.f_{\text {Right }}\right)$ determines the boundary condition at the left (right) side of the trap center, i.e. $x=-L / 2(x=L / 2)$, for any other space location in the trap the function is determined by the fDefault. It is important to remember that the boundary conditions are needed in order to ensure a vanishing probability outside the trap.

We use 4th-order Runge-Kutta method to solve the equation (15) whose solution for one time-step $(\Delta t)$ is approximated by,

$$
\psi(t+\Delta t) \sim \psi(t)+\frac{\Delta t}{6}\left(k_{1}+2 k_{2}+2 k_{3}+k_{4}\right)
$$

The functions ki with $i \in[1,4]$ are defined as,

$$
\begin{gathered}
k_{1}=f(x, t, \psi), \\
k_{2}=f\left(x, t+\frac{\Delta t}{2}, \psi+k_{1} \frac{\Delta t}{2}\right), \\
k_{3}=f\left(x, t+\frac{\Delta t}{2}, \psi+k_{2} \frac{\Delta t}{2}\right), \\
k_{4}=f_{4}\left(x, t+\Delta t, \psi+k_{3} \Delta t\right) .
\end{gathered}
$$


We implement a single-core (serial) code using $\mathrm{C} / \mathrm{C}++$ language and Boost uBLAS libraries.

In all calculations, we set the dimensionless kinetic and repulsion interaction energies as $\epsilon=1$ and $g=$ 1. The spread of the initial Gaussian profile is $\sigma=0.75$ and the amplitude of the trapping potential is $A=15 / 8$, these values are chosen to allow an adiabatic evolution in the initial single-well trap. The spaceand time-steps are set to $\Delta x=0.1$ and $\Delta t=0.001$.

\section{Numerical results}

\section{5.a. Adiabatic regime}

When the dynamical driving is performed sufficiently slow, the BEC has enough time to adapt to the changes of the trapping potential, this regime is known as the adiabatic regime. In this limit, the external trap works as a perfect waveguide as it is shown in figure 3, where the evolution of the density is presented for the symmetric double-well potential in panel 3(a) and the Mach-Zehnder potential in panel 3(b). For both cases the 50:50 beam splitter is simulated by the spatial separation of the initial wave-packet into two well separated matter-waves which later on are smoothly recombined. In the symmetric case, the recombination is perfect and the matter-wave center of mass nicely follows the minimum of the trapping potential. On the other hand, enlarging one arm of the waveguide, it is possible to observe in figure 3(b) that particles gained some extra kinetic energy presenting wave oscillations around the potential minimum after the recombination point.
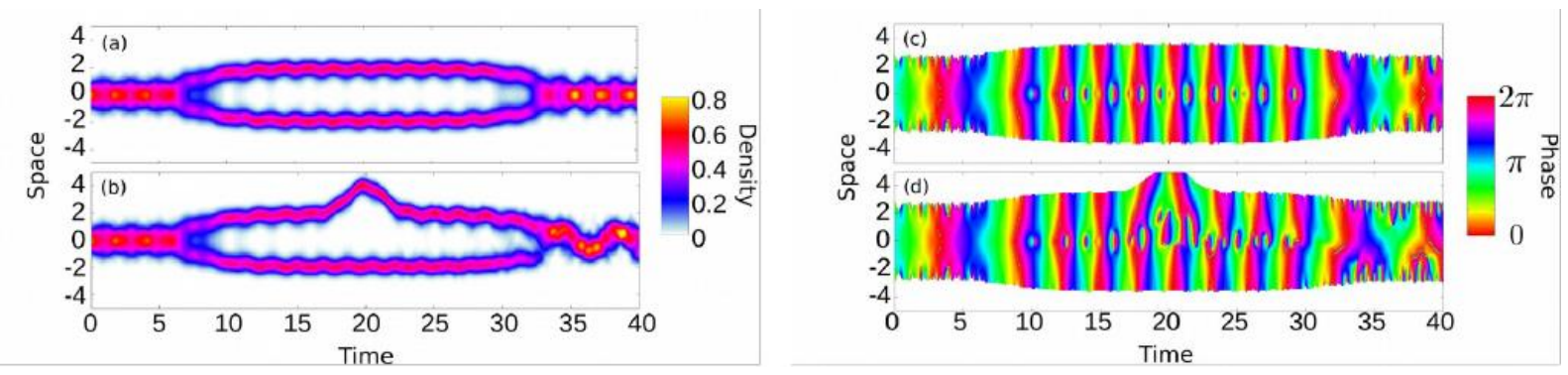

Fig.3. (Color online) Matter-wave interferometer in the adiabatic regime. Panels: density (a) and phase (c) using the symmetric trapping potential. Density (b) and phase (d) using the non-symmetric trap.

It is interesting to have a look at the phase of the propagated matter-wave. As before, the symmetric doublewell is presented in figure 3(c) and the Mach-Zehnder double-well in panel 3(d). Note the highly-symmetric phase-pattern before the splitting and after the recombination, in panel the (c), that shows a perfect phase match of both separated matter-beams. While for the non-symmetric trap, there is a phase difference explicitly observed around $t=20$. Therefore, from this point on the phases registered in both arms differ from each other and the pattern shifts after the recombination as well.

\section{5.b. Adiabatic splitter and sudden recombiner}

The opposite limit for the adiabatic regime is achieved when there is a sudden change of parameters or quench. In this section, we are interested to make first an adiabatic beam splitting followed by a quench at the recombination point by abruptly changing the double-well potential to a single-well trap. Figure 4 shows the evolution of the population and the phase for this specific case. The motivation to study such traps is that the sudden recombination of the two beams in an interferometer is used to map the phase difference into the density when there is a lack of mechanism to measure such physical quantity [13]. Figure 4(a) shows the population as a function of time in the absence of the phase-shifter with a perfect recombination and a very symmetric interference pattern with respect to the $\mathrm{x}=0$ horizontal line, evidenced by the bright points where the density gathers together lying on this line. In figure 4(b), the recombined density pattern shows asymmetries with respect of $x=0$ line in the quantum carpet, reflecting the introduced phase difference. This pattern also shows bright points moving following a zigzag from side to side during the recombination process. Figure 4(c) shows a nice symmetric phase evolution displayed a rhomboid pattern. Whereas the introduced phase shift, placed around $t=20$, develops a highly asymmetric interference with 
respect of the horizontal line after the recombination, although it reveals an emerging zigzag pattern towards the end of the evolution as shown in figure 4(d).
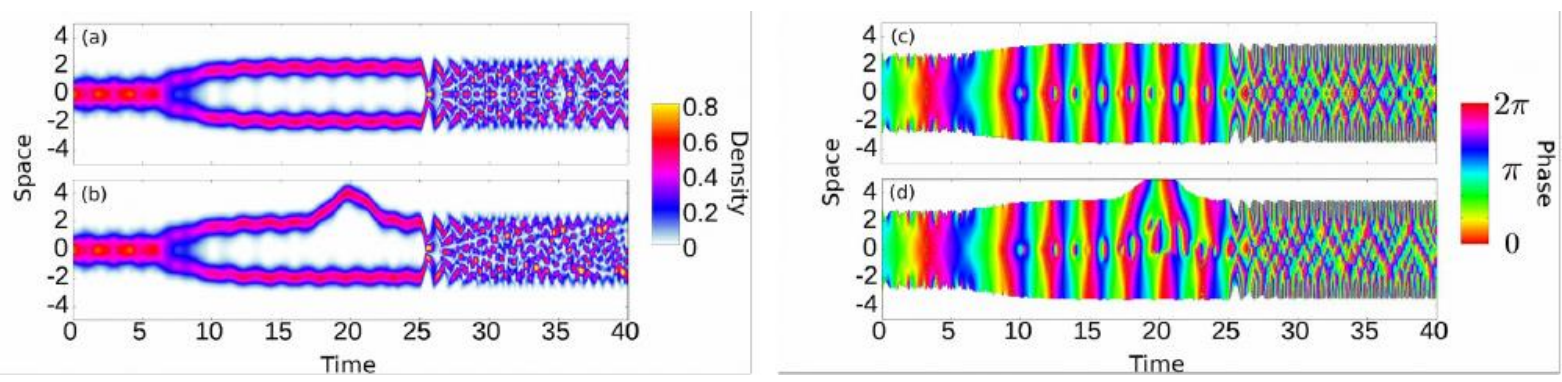

Fig.4. (Color online) Matter-wave interferometer in the case of an adiabatic splitting and a sudden recombination. Panel labelling is the same as in figure 3.

\section{5.c. Non-Adiabatic regime}

Finally, let us discuss the case where there are two quenches performed in the evolution, one at the splitting point and the other at the recombination one. In this case, the whole propagation falls inside the nonadiabatic regime and the corresponding results are presented in figure 5. One can observe that when no phase shifting has been introduced the density wave-function gets distorted although the waveguide is still functional, see figure 5(a). On the other hand, when one path length is enlarged the wave-function not only gets distorted but the fluctuations are so strong that the wave-package struggles to remain in the trap. Density loses can be observed in the left hand side of the propagation direction, see figure 5(b).
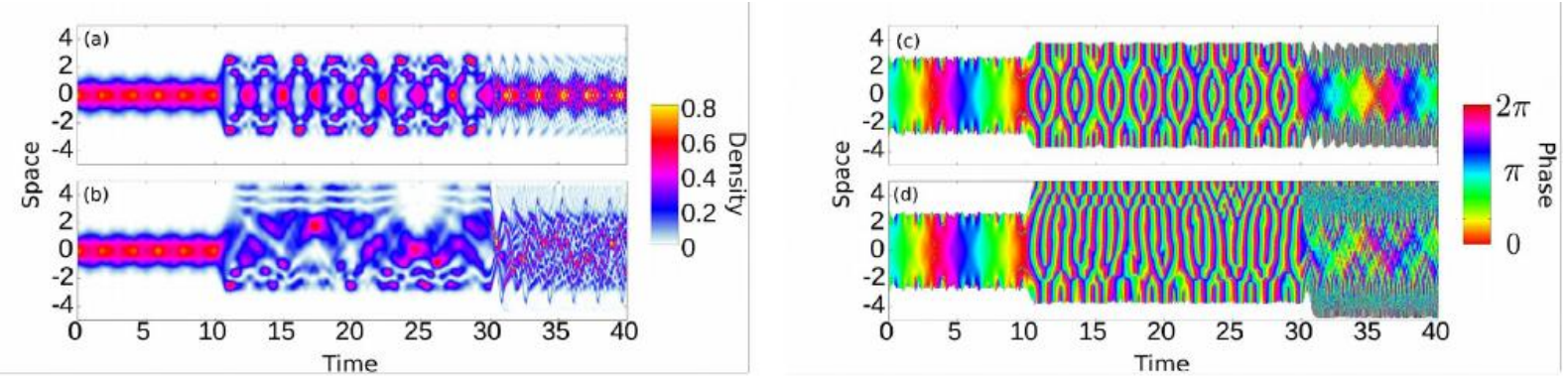

Fig.5. (Color online) Matter-wave interferometer in the case of sudden splitting and sudden recombination. Panel labelling is the same as figure 3.

The non-adiabatic propagation of the phase is presented in figures 5(c) and 5(d). Panel (c) shows the phase evolution for the symmetric trap reflecting the nice waveguide character of the trap despite the sudden change. The retrieved results using the Mach-Zehnder trap are presented in figure 5(d), where it is still possible to observe a clear phase pattern with the acquired phase shift due to the path length difference although the density is quenched. Further on, the waveguide gets so distorted that the results are no longer clear after the recombination point.

\section{Conclusions}

We implement a numerical solution to analyze the propagation of a Bose-Einstein Condensate on a timedependent external trapping potential. We show that the trapping behaves as a perfect waveguide when the dynamical changes are performed slow enough, i.e. in an adiabatic way. On the other hand, when facing sudden changes, the excess of kinetic energy dominates the system dynamics and leads to strong density oscillations. Therefore, the waveguide is distorted and there is a wave-mixing between the two beam branches. Due to the length difference among the two beam paths, there is an extra acquired phase allowing the system to be analyzed as a matter-wave Mach-Zehnder interferometer. The use of two well-known numerical methods, namely finite-difference and Runge-Kutta methods, reduces the numerical complexity implementation. These tools allow us to solve the 1D Gross-Pitaevskii equation which is very popular nowadays due to the growing interest on ultracold matter system in the last decades. Hence, this kind of work can be proposed as exercise in standard courses of quantum mechanics and computational physics involving state of the art developments. 


\section{Acknowledgements}

This work has been supported by Universidad del Valle under the internal projects 71107. The authors acknowledge the Excellence Center of New Materials (CENM) Gran no. RC275-2011 for financial support of the research group. 